\title{
Le test de concordance de script (TCS) comme outil d'évaluation formative des internes en neurochirurgie: implantation du test sur Internet à l'échelle nationale
}

\author{
François CAIRE*, Jean-Christophe SO ** , Bernard CHARLIN** , Philippe ISIDORI ****, \\ Jean-Jacques MOREAU*
}

\begin{abstract}
Résumé Contexte : Le text de concordance de script (TCS) a éé choisi par le Collège des enseignants de neurochirurgi e comme outil d'auto-évaluation desinternes. Jusqu'à présent, ceux-ci ne disposaient en effet d'aucun système d'éraluation formative leur permettant de faire le point sur leurs forces et leurs faiblesses en cours de formation. But : Rapporter les étapes de mise en place du TCS pour l'auto-évaluation de l'ensemble desinternes en formation. Le test et accessible sur Internet dans le cadre du campus numérique de neurochirurgie L' administration régulière de tests permettra à chacun de suivre l'évolution de ses résultats au cours de sa formation. Mise en place : La rédaction d'une banque de questions a débuté, tous les domaines de la spécialité n'étant pas encore couverts Le logiciel permettant l'informatisation des testsa par ailleurs été mis au point, permettant la réalisation d'une première évaluation par TCS au cours des «D euxièmes Journées de N eurochirurgie » en mars 2003. Conclusion : Les internes en neurochirurgie dis posent donc désormais d'un mode d'auto-évaluation facilement access ble pui squeinformatisé. Les efforts porteront désormaissur la diffusion de cet outil et sa pérennisation.
\end{abstract}

Mots clés Internes; neurochirurgie; évaluation ; évaluation formative; raisonnement clinique; incertitude; problème mal structuré; test de concordance de script.

Summary Background: No effective tool was up to now available for formative assessment of neurosurgery residents during their training. The script concordance tet (SCT) was therefore chosen by the Collège des Enseignants to allow them to assess themselves ther clinical competence Aim: The aim of this work is to describe the constitution of a data bank available for further building of script concordance tests The test is available on the web. The evolution of the results of each student will be followed up by regular assessment during the residency. M ethod: A first series of about 200 tests was created between may and novembre 2002, covering the main fields of neurosurgery. The first assessment of neurosurgery residents by script concordance test was carried out in march 2003, during the second "Journées de Neurochirurgie». Conclusion: A new tool isnow available for auto-assessment of neurosurgery residents, easy to use on the web. Diffusion to other branches and perennity of this work are now our main aims.

Key Words N eurosurgery; internship; residency; assessment; formative assessment; clinical reasoning; uncertainty; ill defined problem ; script concordance test.

Pédagogie M édicale 2004 ; 5 : : 87-94

* Service de neurochirurgie, CHU Dupuytren, 2 avenue Martin Luther King, 87042 Limoges

** Service de neurochirurgie, OHU de Rangueil, 2 avenue Jean-Poulhès, 31400 Toulouse

** * URDESS, université de Montréal, facultéde médecine, CP 6128 succursale centre ville, Montréal, Québec, Canada H3C3J7

**** DCAM, université Victor Segalen Bordeaux II, 146 rue Léo Sajgnat, 33076 Bordeaux cedex

Correpondance: F. CAIRE, service de neurochirurgie, CHU Dupuytren, 2 avenue Martin Luther King, 87042 Limoges, France. Téléphone: 05.55.05.65.21 - mailto:fpcaire@yahoo.fr 


\section{Concepts et Innovations}

\section{Introduction}

L'évaluation formative a un rôle important à jouer dans l'acquisition des compétences médicales. Loin des modalités classiques d'évaluation sanctionnelle, elle a une véritable fonction d'apprentissage: elle permet à l'apprenant de percevoir ses forces et ses faiblesses et favorise ainsi l'autonomie dans les apprentissages.

L'organisation actuelle des cursus de troisième cycle spé cialisés en Franceneprévoit pas d'évaluation formative des internes au cours de leur formation. Certes, l'évaluation est en fait quotidienne et formalisée en fin de semestrepar une synthèse réali séé par le chef du service d'accueil. $M$ ais, dans cette situation, l'interne ne dispose d'aucun moyen pour mesurer ses propres compétences et leur évolution au cours de sa formation. C'est pour combler cemanque que le Collège des enseignants en neurochirurgie a décidé la mise en place d'une évaluation formative des internes de la spécialité au cours de leur cursus.

L'outil retenu a étéle test de concordance de script (TCS). II sagit d'un nouveau mode d'évaluation, conçu pour explorer une des dimensions de la compétence médicale: le raisonnement clinique. Ses applications possibles sont diverses, de l'évaluation des internes à la formation médicale continue.

Les contraintes spécifiques de formation en neurochirurgie, telles que le petit nombre d'internes en formation et leur grande dispersion géographique (moins d'une centaine d'internes pour l'ensemble des $\mathrm{CH}$ U ), ont par ailleurs conduit à utiliser le réseau internet, par l'intermédiai re du sitedu campus numérique de neurochirurgie, pour mettre en place cette nouvelle évaluation.

L'article décrit les étape suivies pour mettre en place l'auto-évaluation des internes en neurochirurgie par TCS, aujourd'hui accessible à tous, avec un identifiant individuel, sur le site internet du campus numérique de neurochirurgie (www.uvp5.univ-paris5.fr/campus-neurochirurgie) et la place qu'elle occupe désormais dans le cursus de neurochirurgie.

\section{Le test de concordance de script (TCS)}

Le test de concordance descript (TCS) permet l'évaluation du raisonnement clinique en contexte d'incertitude. II compare les réponses données par la personne qui passe le test à celles données par un groupe d'experts de référence. II n'a donc pas vocation à remplacer des modes d'évaluation déà existants - et éprouvés - comme les $Q C M$, qui explorent bien les connaissances liées aux problèmes cli- niques bien définis. II vient plutôt en complément, comme moyen d'approcher le raisonnement dans des problèmes mal définis, c'est-à-dire lorsque toutes les données nécessaires à la résolution du problème ne sont pas présentes ou Iorsqu'il existe des données ambiguës ${ }^{1,2}$.

D ans un TCS, il n'est pas demandé à l'étudiant de réciter la liste des diagnostics possibles, mais de fairela synthèse de ses connaissances dans une situation clinique donnée afin de proposer un diagnostic ou/et une stratégie de prise en charge. Le principe du test a déjà éééexposé précédemment par son concepteur ${ }^{3,4,5}$. N ous rappellerons simplement que le TCS est un examen constitué d'un nombre variable d'unités. C hacune de ces unités a pour base une courte vignette décrivant une situation clinique classique susceptible d'être rencontréeen pratiquecourantepar leclinicien. La situation clinique décrite pose un problème, même pour un expert du domaine, dans la mesure où il manque un ou plusieurs éléments pour que l'on puisse proposer de façon certaine un diagnostic ou une stratégie de prise en charge (diagnostique ou thérapeutique). Certaines données peuvent également être ambiguës et difficilement interpré tables. Sont ensuite proposées différentes hypothèses de diagnostic ou de prise en charge puis, pour chacune, est proposé un nouvel élément clinique ou paraclinique, dont on évalue l'impact sur la valeur de l'hypothèse de départ, mesuré sur une échelle de Likert à cinq entrées.

Letableaul est un exemple d'unité proposée à des internes de neurochirurgie.

D ifférents formats sont possibles. Ils peuvent concerner le choix d'examens complémentaires ou des options de prise en charge thérapeutique. Dans une unité diagnostique, une hypothèse diagnostique est proposée dans la première colonne, tandis qu'une donnée clinique ou paraclinique susceptible de modifier la valeur de cette hypothèse est proposée dans la deuxième colonne.

On notera que la signification des valeurs numériques de l'échelle de Likert varie selon les formats: -2 peut, par exemple, signifier «l'hypothèse est pratiquement éliminée » ou «l'examen est formellement contre-indiqué » sel on le format de l'unité (ici diagnostic ou investigation). $L^{\prime}$ autre aspect très novateur du TCS tient dans le mode de correction : il n'y a pas en effet une seule bonne ou mauvaise réponse. La grille de correction est établie par un groupe d'experts qui, plutôt que de s'entendre sur une réponse consensuelle, complètent individuellement le test. Le crédit accordé à chacune des réponses possibles $(-2,-1$, $0,+1,+2)$ est calculé selon le nombre d'experts qui l'ont choisie: on divise le nombre d'experts qui ont 


\section{Tableau 1 : \\ Exemple de vignette clinique élaborée pour construire un test de concordance de script en neurochirurgie}

Vous voyez en consultation un homme de 59 ans, maçon, pour des troubles de la marche saggravant progressivement depuis six mois. II invoque une chute sur son lieu de travail survenue un an auparavant. II a également noté depuis quatre semaines des douleurs irradiant au membre inférieur gauche Vous notez à l'examen la présence d'un steppage à gauche.

\begin{tabular}{|l|l|r|}
\hline Si vous pensiez à : & Et qu'alors vous trouvez : & $\begin{array}{l}\text { L'effet sur votre hypothèse } \\
\text { diagnostique est le suivant : }\end{array}$ \\
\hline $\begin{array}{l}\text { Atteinte du nerf } \\
\text { fibulaire au genou }\end{array}$ & $\begin{array}{l}\text { H ypoesthésie de la loge } \\
\text { antéro-externe de } \\
\text { la jambehomo-latérale }\end{array}$ & $-2 \quad-1 \quad 0 \quad+1 \quad+2$ \\
\hline $\begin{array}{l}\text { Conflit disco- } \\
\text { radiculaire }\end{array}$ & $\begin{array}{l}\text { Signe de Lasègue } \\
\text { homolatéral à 15 }\end{array}$ & $-2 \quad-1 \quad 0 \quad+1 \quad+2$ \\
\hline $\begin{array}{l}\text { Canal lombaire } \\
\text { étroit }\end{array}$ & $\begin{array}{l}-2 \\
\text { Syndrome pyramidal } \\
\text { aux membres inférieurs }\end{array}$ & $-1 \quad 0 \quad+1 \quad+2$ \\
\hline
\end{tabular}

Entourez la proposition qui vous semble adéquate :

-2 I'hypothèse est pratiquement éliminée

-1 l'hypothèse devient moins probable

0 l'information n'a aucun effet sur l'hypothèse

+1 I'hypothèse devient plus probable

+2 il ne peut sagir pratiquement que de cette hypothèse

choisi cette réponse par le nombre d'experts ayant opté pour la réponse la plus choisie. Ainsi, pour un groupe de 10 experts, s 8 choisissent « -2 » et 2 la réponse « $-1 »$ la valeur de la réponse « -2 » sera $8 / 8=1$ point, et celle de la réponse $\ll-1 »$ sera $2 / 8=0,25$.

L'absence de «bonne réponse » tient à une raison simple: le TCS est destiné à évaluer la capacité de l'étudiant à résoudre des situations dans lesquelles une telle réponse n'existe pas, plusieurs interprétations étant recevables. II reflète la capacité de l'étudiant à faire face à une situation courante dans laquelle il existe un certain degré d'incertitude, en formulant des hypothèses et en évaluant l'impact de nouvelles données sur le statut de ces hypothèses.

Le TCS reflète donc avant tout la capacité à résoudre des problèmes cliniques mal définis ( « ill-defined problems»). 


\section{Concepts et Innovations}

\section{Les TCS en neurochirurgie}

La mise en place desTCS en neurochirurgie a été décidée par le Collège des enseignants après l'analyse des résultats d'un questionnaire portant sur les besoins ressentis par les internes en matière d'enseignement. C equestionnaire leur avait été soumis lors des journées nationales d'enseigne ment de Saint-M alo en novembre 2001. L'analyse des résultats montrait d'une part, le besoin pour les étudiants d'une harmonisation de l'enseignement au niveau national et d'autre part, une inquiétude quant à leurs difficultés à évaluer leur niveau, tant par rapport aux autres internesque par rapport au niveau de connai ssances requis en fin d'internat. En effet, la neurochirurgie est caractérisée par le peit nombre d'internes engagés dans le cursus (une cinquantaine), ainsi que par leur grande dispersion géographique. En réponse à cette analyse de besoins, le Collège des enseignants de neurochirurgie a pris la décision d'utiliser le TCS comme outil d'auto-évaluation des internes. Le test est destiné à leur permettre d'évaluer leur propre niveau dans les différents domaines de la neurochirurgie ainsi que leur progression et l'évolution de leurs résultats. Sont concernés les étudiants de troisième cycle spécialisé en neurochirurgie: diplôme d'éudes spécialisées (DES), Diplôme inter-universitaire de spécialité (DIS), mais aussi des étudiants étrangers en attestation de formation spécialisée (AFS).

L'importance d'une évaluation de type formatif au cours de l'apprentissage est maintenant bien connue ${ }^{6,7,8}$. C'est cette fonction que revêt leTCS en neurochirurgie : il permet à l'interne, sous la supervision de son tuteur, de mesurer ses points forts, ses faiblesses, et de guider en consé quence ses apprentissages. Tel qu'il est utilisé, le test ne constitue donc pas une évaluation sanctionnelle; il ne sagit en aucun cas d'un examen de passage

L'intérêt principal du TCS dans ce cadre est de permettre l'évaluation du raisonnement clinique, donc de se situer à un niveau taxonomique plus élevé que la plupart des modes d'évaluation existants. II est, par ailleurs, simple à mettre en place pour un groupe relativement important (une centaine d'étudiants).

II a été décidé de soumettre aux étudiants desT CS constitués de trente unités, une unité représentant un cas clinique à trois questions (items pour les docimologues) avec un temps de passage de soixante minutes, ce qui ramène à deux minutes en moyenne le temps passé par unité. L'administration du test par voieinformatique sur internet a été choisie pour des raisons pratiques: l'éloignement géographiquerend, en effet, illusoireuneévaluation nationaleà une même dateet dans un mêmelieu. Internet offre un moyen simple de contourner cet obstacle.

Un logiciel conçu spécialement pour les TCS a été développé par le département communication, audiovisuel et multimédia (D CAM ) de l'université Bordeaux II. C elogiciel est accessible par un portail internet. Le coordonna teur a la possibilité de créer un test lorsqu'il le désire, en utilisant des unités présentes dans une banque. II peut sélectionner les unités sel on différents critères ou de façon aléatoire. Chaque interne dispose d'un mot de passe lui permettant d'accéder au site et de passer le test dans le délai imparti.

Les internes passent le test une fois par trimestre. Le site reste accessible pendant plusieurs jours, ce qui laisse à chacune une certaine liberté d'organisation, vis-àvis notamment des gardes. Après son passage, chaque interne accède à ses résultats bruts. Enfin, après la date finale de passage, il a accès aux résultats «travaillés » : résultats globaux des autres internes, résultats par promotion, etc.

Il est nécessaire de n'offrir qu'une plage de temps limitée à quelques jours pour le passage du test. En effet, cela permet d'exprimer les résultats d'un individu par rapport à ceux de l'ensemble du groupe d'étudiants ayant passé le test (évaluation à interprétation normative).

\section{Apports d'une évaluation par TCS en neurochirurgie}

\section{Bénéfices individual et pédagogique}

LeTCS en neurochirurgie est destinéà uneévaluation formative des internes, et non à une évaluation sanctionnelle. II ne sera en aucun cas utilisé dans un but de validation ou de non validation du cursus.Comme nous l'avons vu, il est possible aux concepteurs du test d'en choisir lecontenu : choix par présentation clinique (si l'on désire évaluer le niveau d'expertise des internes quant aux paraplégies post-traumatiques par exemple) ou par domaine (rachis dégénératif, tumeurs médullaires, etc). L'étudiant peut ainsi évaluer ses forces et ses faiblesses de façon détaillée et recentrer si besoin ses apprentissages. Par ailleurs, la possibilité pour l'interne de suivre ses propres résultats au cours de son cursus apporte un aspect dynamique à cette évaluation.

Les résultats du test sont exprimés de façon classique par une note. Toutefois, les résultats «bruts », chiffrés selon la grille de correction des experts, qui donnent donc un score 
par rapport aux réponses des experts, n'ont qu'un intérêt limité

Le passage du test par un large groupe d'étudiants permettra, en revanche, à l'interne, outre ces résultats bruts, de se situer par rapport à des internes de même niveau ou d'autres niveaux (internes plus ou moins avancés dans leur cursus). Toutefois, ceci suppose que la totalité des étudiants (inscrits au DES ou au DIS et étudiants étrangers) se soumettent au TCS de façon régulière, dans un cadre qui sera nécessairement formalisé.

La réroaction est également le fait du tuteur (un enseignant, choisi par l'étudiant en début d'internat pour le suivre sur le plan pédagogique). Celui-ci a en effet pour fonction d'analyser avec l'interne ses résultats, afin de l'aider à en retirer un bénéfice maximal.

\section{Bénéfice institutionnel}

Sur le plan interrégional et national, l'analyse globale des résultats des internes permettra de vérifier l'adéquation des programmes d'enseignements au niveau de connaissances des étudiants, et de recentrer le cas échéant ces programmes.

\section{Démarche de mise en place}

Le travail de mise en place d'une auto-évaluation des internes par TCS en neurochirurgie a débuté en janvier 2002. Au cours d'une réunion du collège des enseignants, les experts présents ont déterminé:

- une liste des trente-deux présentations cliniques les plus représentatives de la spécialité. Ces présentations ont été sélectionnées sel on la règle «PUIGE » (prévalence, urgence, intervention possible, gravité, exemplarité). II pouvait sagir de situations cliniques rencontrées en consultation ou en hospitalisation, en urgence ou non. Les présentations rares ou inhabituelles ont volontairement été écartées.

- une liste des trois ou quatre options les plus pertinentes pour chacune de ces situations, ainsi que les principaux éléments cliniques ou paracliniques à rechercher.

Ultérieurement a été créée une liste de « domaines » cor-

\begin{tabular}{|c|c|}
\hline \multicolumn{2}{|c|}{$\begin{array}{l}\text { Tableau } 2 \text { : } \\
\text { G rille de correction utilisée par les neurochirurgiens experts } \\
\text { pour la validation des unités du test de concordance de script }\end{array}$} \\
\hline \multirow[t]{3}{*}{$\begin{array}{l}\text { La vignette clinique } \\
\text { (énoncé du problème) }\end{array}$} & $\begin{array}{l}\text { La vignette est-elle correctement rédigée? } \\
\text { (incorrections, longueur, confusion) }\end{array}$ \\
\hline & $\begin{array}{l}\text { La vignette correspond-elleà } \\
\text { une situation clinique crédible? }\end{array}$ \\
\hline & $\begin{array}{l}\text { S'agit-il d'une présentation clinique usuelle? } \\
\text { (la situation ne doit pas être un piège) }\end{array}$ \\
\hline \multirow[t]{3}{*}{$\begin{array}{l}\text { Les items } \\
\text { (individuellement) }\end{array}$} & $\begin{array}{l}\text { Les hypothèses diagnostiques (ou les options } \\
\text { de prise en charge) qui sont proposées sont-elles pertinentes? }\end{array}$ \\
\hline & $\begin{array}{l}\text { Les items correspondent-ils à des questions que } \\
\text { I'on se pose réellement en situation clinique? }\end{array}$ \\
\hline & $\begin{array}{l}\text { Le niveau de difficulté correspond-il } \\
\text { à ce qu'un interne devrait savoir? } \\
\text { (item trop facile ou trop difficile) }\end{array}$ \\
\hline
\end{tabular}




\section{Concepts et Innovations}

\section{Tableau 3 : \\ Travail correctif de validation des unités du test de concordance de script (TCS), réalisé par les neurochirurgiens experts}

\section{a) Exemple d'unité de TCS avant correction}

M adame Y., 65 ans, a depuis plusieurs mois d'importants troubles de la marche. Elle se plaint de manquer de force dans les jambes; son périmètre de marche est limité. Vous notez dans ses antécédents un surpoids (80 kg pour $1,65 \mathrm{~m}$ ), une cure chirurgicale de reflux gastro-œsophagien il y a dix ans.

\begin{tabular}{|c|c|c|}
\hline Si vous pensiez à & Et qu'alors vous trouvez & $\begin{array}{l}\text { L'effet sur votre hypothèse } \\
\text { diagnostique est le suivant }\end{array}$ \\
\hline Canal lombaire étroit & $\begin{array}{l}\text { Atteinte motrice pure, } \\
\text { sans troubles sensitifs à l'examen }\end{array}$ & $\begin{array}{llll}-2 & -1 & 0 & +1\end{array}$ \\
\hline Pseudo-myopathie iatrogène & Traitement substitutif de la ménopause & $\begin{array}{lllll}-2 & -1 & 0 & +1 & +2\end{array}$ \\
\hline M yélopathie cervicarthrosique & Amyotrophie quadricipitale & $\begin{array}{llll}-2 & -1 & 0 & +1\end{array}$ \\
\hline
\end{tabular}

Entourez la proposition qui vous semble adéquate :

-2 l'hypothèse est pratiquement éliminée

-1 l'hypothèse devient moins probable

0 l'information n'a aucun effet sur l'hypothèse

+1 l'hypothèse devient plus probable

+2 il ne peut sagir pratiquement que de cette hypothèse

b) Unité de TCS précédente après correction par les experts

$M$ adame Y., 65 ans, a depuis plusieurs mois d'importants troubles de la marche. Elle se plaint de manquer de force dans les jambes; son périmètre de marche est limité. Vous notez dans ses antécédents un surpoids (80 kg pour $1,65 \mathrm{~m}$ ), une cure chirurgicale de reflux gastro-œsophagien il y a dix ans.

\begin{tabular}{|c|c|c|c|c|c|c|}
\hline Si vous pensiez à & Et qu'alors vous trouvez & \multicolumn{5}{|c|}{$\begin{array}{l}\text { L'effet sur votre hypothèse } \\
\text { diagnostique est le suivant }\end{array}$} \\
\hline C anal lombaire étroit & $\begin{array}{c}\text { Atteinte motrice pure, } \\
\text { sans troubles sensitifs à l'examen }\end{array}$ & -2 & -1 & 0 & +1 & +2 \\
\hline $\begin{array}{l}\text { Artériopathie oblitérante } \\
\text { des membres inférieurs }\end{array}$ & D ysesthésies et douleurs nocturnes & -2 & & 0 & +1 & +2 \\
\hline M yélopathie cervicarthrosique & Amyotrophie quadricipitale & & 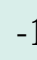 & 0 & +1 & +2 \\
\hline
\end{tabular}

Entourez la proposition qui vous semble adéquate :

-2 I'hypothèse est pratiquement éliminée

-1 l'hypothèse devient moins probable

0 l'information n'a aucun effet sur l'hypothèse

+1 I'hypothèse devient plus probable

+2 il ne peut s'agir pratiquement que de cette hypothèse 
respondant aux différentes sous-spécialités individualisables en neurochirurgie: dégénératif, neurochirurgie pédiatrique, neurochirurgie fonctionnelle, etc. Entre mai et octobre 2002, deux des auteurs, boursiers du campus numérique de neurochirurgie, ont procédé à la rédaction d'une première série de 222 unités, dans le cadre d'un préceptorat de pédagogie réal isé à l'U nité de Recherche et de D éveloppement en Education des Sciences de la Santé (U RDESS) à I'U niversité de M ontréal.

Les principales difficultés rencontrées lors de la rédaction de ces premières unités sont détaillées ailleurs ${ }^{9}$. Elles portent sur : a) la rédaction de chaque vignette : il ne doit pas sagir d'un cas inhabituel ou très atypique. Par ailleurs, la formulation doit ouvrir la voie à plusieurs options de diagnostic ou de prise en charge de pertinence équivalente (puisqu'il s'agit de problèmes mal définis) ; b) l'estimation du degré de difficulté, les outils docimologiques sappliquant mal aux TCS ; c) la sélection de problèmes spécifiquement neurochirurgicaux, le risque étant de privilégier les très nombreux diagnostics différentiels neurologiques ou rhumatologiques.

Les unités rédigées al ors ont été soumises pour correction et validation à un nouveau groupe d'experts, chacun recevant un groupe d'une vingtaine d'unités. Cette étape de validation était réalisée à l'aide d'une grille (tableau 2), indiquant aux experts les principaux éléments à vérifier au cours de cette étape.

Le tableau 3 ( $a$ et b) illustre l'évolution d'une unité avant et après correction. Les remarques formulées par les correcteurs portaient ici sur le contenu du deuxième item : le diagnostic proposé ne correspondait pas à une des trois hypothèses les plus probables dans un tel contexte. Les propositions faites étaient : maladie de C harcot et artériopathie oblitérante des membres inférieurs.

D ifférents signes cliniques à rechercher étaient également indiqués.

Le processus que nous avons décrit représente le début de la réalisation d'une vaste banque d'items. Les unités rédigées à ce jour ne couvrent qu'une part de la spécialité: si toutes les présentations cliniques ont été abordées, en revanche certains domaines n'ont fait l'objet d'aucune unité. Par ailleurs, la majorité des unités rédigées sont de type diagnostique

D e larges champs restent donc à explorer, notamment la création d'unités de type thérapeutique (exploration des conduites à tenir sur le plan chirurgical, en particulier), ainsi que d'unités faisant appel à l'interprétation en imagerie.
Enfin, en janvier 2003, le collège des enseignants de neurochirurgie a réuni 20 experts chargés de l'établissement de la grille de correction des vignettes corrigées. II sagissait de neurochirurgiens «seniors ». Ils ont pu être répartis en trois groupes, chacun établissant la grille de correction d'une série de soixantequatorze unités. Au cours de cette réunion a pu être présenté et testé le logiciel développé par le D CAM.

\section{Première administration du test}

II n'existait pas d'obstacle institutionnel, en raison de l'implication du collège des enseignants de neurochirurgie dans le projet. L'existence du campus numérique de neurochirurgie a par ailleurs offert une « coquille», au sein de laquelle l'évaluation par TCS a pu prendre naturellement sa place.

Un premier essai d'évaluation par TCS a pu être réalisé à l'occasion des deuxièmes journées de neurochirurgie organisées par la Société française de neurochirurgie à Perpignan du 26 au 28 mars 2003. Deux postes informatiques dotés d'un accès à internet ont été mis à la disposition des internes. Ceux-ci ont reçu à leur inscription un mot de passe permettant un accès protégé au site, ainsi qu'un document explicatif. La connection était possible pendant toute la durée du congrès.

LeTCS proposé était constitué de trente unités (vignette +3 items) avec une durée de passage d'une heure. Les différentes unités ont été scindées en trois groupes de 10 unités selon les domaines correspondants: neurochirurgie tumorale, rachis dégénératif et neurochirurgie vasculaire. Les objectifs principaux de ce premier essai ont été atteints, puisque nous avons pu :

- vérifier le bon fonctionnement du logiciel mis au point par le DCAM

- sensibiliser les internes présents à cet outil par une pré sentation faite au cours du congrès et par un passage du test par chacun d'eux sur les ordinateurs mis à leur disposition

- recueil lir les réactions des utilisateurs, un questionnaire leur ayant été envoyé par la suite. L'acceptabilité du test semble bonne, aucune critique n'ayant éé relevée tant sur le principe du test que sur son mode de présentation informatique. 


\section{Letest de concordance de script (TCS)...}

\section{Conclusion}

L'outil choisi pour permettre l'auto-évaluation des internes en neurochirurgie au cours de leur cursus de spé cialité est le test de concordance de script. Le travail déjà réal isé à ce jour a permis de poser les bases de cette évaluation: information des étudiants et des enseignants, création d'une première banque de question et mise au point du logiciel qui permet d'informatiser lestests. L'outil testé avec succès en mars 2003, a été développé dans le cadre du campus numérique de neurochirurgie qui permet désormais l'accès aux évaluations par TCS.

II importe donc maintenant de se consacrer à deux objectifs essentiels: il sagira d'une part, de sensibiliser les internes à cet outil qui est désormais à leur disposition et d'autre part, de former des enseignants qui pourront prendre part à la rédaction de nouvelles unités, afin d'entretenir la banque de questions et de pérenniser cetteinnovation. Enfin, le travail réalisé en neurochirurgie pourra facilement être diffusé à d'autres spécialités. N ous avons pour cela essayé de faire part des enseignement issus de notre expérience ${ }^{9}$. Des contacts ont également été pris avec des enseignants d'autres spécialités intéressés par I'utilisation des tests de concordance de script.

\section{Remerciements}

Ce travail a éé rendu possible par deux bourses du campus numérique de neurochirurgie aux auteurs.

\section{Références}

1. Llorca G, Roy P, RicheB. Evaluation de la résolution de problèmes cliniques mal définis en éthique dinique: variation des scores selon les méthodes de correction et les caractéristiques des jurys. Pédagogie médicale $2003 ; 4$ : 80-88.

2.Charlin B, van der Vleuten C. Standardized assessment in context of uncertainty : the script concordance approach. Eval Health Prof, submitted, july 2003.

3. Charlin B, Tardif J, Boshuizen H P. Scripts and medical diagnostic knowledge theory and applications for dinical reasoning instruction and research. Acad M ed 2000 ; 75 : 182-190.

4. Charlin B, Roy L, Brailovski C, Goulet F, van der Vleuten $C$. The Script Concordance tet : a tool to assess the reflective dinician. Teach Learn M ed 2000 ; 12 : 189-195.
5. Charlin B,Gagnon R, Sibert L, Van der Vleuten C. Le test de concordance de script, un instrument d'évaluation du raisonnement dinique Pédagogiemédicale $2002 ; 3$ : 135-144.

6. Crossey J, H umphris G, Jolly B. Assessing health profes sonals M ed Educ $2002 ; 36: 800-805$.

7. WassV, van der Vleuten $C$, Shatzer $J$, Jones R. Assessment of dinical competence Lancet $2001 ; 357$ : 945-949.

8. Jouquan J. L'évaluation des apprentissages des éudiants en formation médicale initiale. Pédagogie Médicale $2002 ; 3: 38-52$.

9. Caire F, Sol JC, M oreau JJ, Is dori P, Charlin B. Autoévaluation des internes en neurochirurgie par tests de concordance de script (TCS) : processus d'élaboration des tests. N eurochirurgie $2004 ; 50: 66-72$. 